\title{
Defective heart development in hypomorphic LSD1 mice
}

\author{
Thomas B Nicholson ${ }^{1,2}$, Hui Su${ }^{1,2}$, Sarah Hevi, ${ }^{1,2}$, Jing Wang ${ }^{2}$, Jeff Bajko ${ }^{2}$, Mei Li ${ }^{1}$, Reginald Valdez ${ }^{1}$, \\ Joseph Loureiro ${ }^{1}$, Xiaodong Cheng ${ }^{3}$, En $\mathrm{Li}^{2}$, Bernd Kinzel ${ }^{4}$, Mark Labow ${ }^{1}$, Taiping Chen ${ }^{1,2,5}$ \\ ${ }^{I}$ Developmental and Molecular Pathways; ${ }^{2}$ Epigenetics Program, Novartis Institutes for BioMedical Research, Cambridge, MA \\ 02139, USA; ${ }^{3}$ Department of Biochemistry, Emory University, Atlanta, GA 30322, USA; ${ }^{4}$ Developmental and Molecular Pathways, \\ Novartis Institutes for BioMedical Research, Novartis Pharma AG Forum 1 Novartis Campus CH-4056, Basel, Switzerland
}

Lysine-specific demethylase 1 (LSD1/AOF2/KDM1A), the first enzyme with specific lysine demethylase activity to be described, demethylates histone and non-histone proteins and is essential for mouse embryogenesis. LSD1 interacts with numerous proteins through several different domains, most notably the tower domain, an extended helical structure that protrudes from the core of the protein. While there is evidence that LSD1-interacting proteins regulate the activity and specificity of LSD1, the significance and roles of such interactions in developmental processes remain largely unknown. Here we describe a hypomorphic $L S D 1$ allele that contains two point mutations in the tower domain, resulting in a protein with reduced interaction with known binding partners and decreased enzymatic activity. Mice homozygous for this allele die perinatally due to heart defects, with the majority of animals suffering from ventricular septal defects. Transcriptional profiling revealed altered expression of a limited subset of genes in the hearts. This includes an increase in calmodulin kinase (CK) 2 3 , the regulatory subunit of the CK2 kinase, which correlates with E-cadherin hyperphosphorylation. These results identify a previously unknown role for LSD1 in heart development, perhaps partly through the control of E-cadherin phosphorylation.

Keywords: epigenetics; LSD1; heart development; E-cadherin

Cell Research advance online publication 6 December 2011; doi:10.1038/cr.2011.194

\section{Introduction}

The development of the mammalian heart is a complex process involving the coordinate interplay of numerous pathways. Because of this, one of the most common causes of lethality in genetically modified mice involves heart defects [1], and congenital heart defects affect approximately $1 \%$ of human newborns [2]. The heart is the first organ to form during development, with differentiation of cardiomyocytes in mice beginning at embryonic day 7.5 (E7.5), leading to the formation of the heart tube. The heart tube begins beating at E8.0, and by E9.0 exhibits a regular beating rhythm [3]. The tube is composed of

Correspondence: Taiping Chen

Tel: +1-512-237-9479; Fax: +1-512-237-2475

E-mail: tchen2@mdanderson.org

${ }^{5}$ Current address: Department of Molecular Carcinogenesis, The University of Texas MD Anderson Cancer Center, Smithville, TX 78957, USA Received 20 April 2011; revised 7 June 2011; accepted 14 October 2011 three layers, the myocardial layer, the endothelial layer, and the cardiac jelly. Recent results have demonstrated that the development of the heart arises from two cell lineages, with the second heart field being the source of the outflow tract and myocardium [4]. The heart tube loops to the right, leading to the eventual formation of the atria and ventricles with contributions from both the first and second heart fields. In the heart, localized "swellings" of the wall appear at approximately E9.5; these are known as cushions, which arise from the cardiac jelly, and are critical for the formation of all septal and valvular structures of the mature heart [5]. The developing heart is initially composed of a single ventricle; subsequent formation of the septum, separating the ventricle into two distinct compartments, allows for the unidirectional flow of blood through the animal. The muscular portion of the ventricular septum begins to appear at E11.5 [3]. The closure of the ventricles is complete by E14.0, at which point the muscular portion, derived from the heart wall, and the membranous portion, arising from the cushions, have fused to form a complete separation between the ventricles [3]. 
Cardiac defects are a major source of late-stage lethality during mouse development, including improper formation of the walls between the chambers, outflow tract malformations, and defects in the cardiac conduction system [1]. The genetics underlying these defects are complex, and may involve large numbers of genes [6]; approximately $80 \%$ of heart defects in human newborns occur in a sporadic manner, with the genetics still being characterized [7]. Because of the complexity of the development of the heart, and the lack of in vitro cellular systems that are available to model the development of the heart, the dissection of these pathways has primarily depended on the characterization of mutant mouse models. For example, the initial identification of the second heart field was with a transgenic mouse expressing lacZ under the control of the $F g f l 0$ gene [8]. The proliferation of cardiac progenitor cells appears to be controlled by a complex interaction between Notch 1 and $\beta$-catenin pathways, and their effects on transcription factors such as Isl1 [9]. Mouse models have since been used to identify many proteins that are involved in the development of the heart (reviewed in [4]). Despite this, much about the molecular mechanisms controlling the development of the heart remains to be clarified.

Human lysine-specific demethylase 1 (LSD1, also known as AOF2/KDM1A) was the first of a group of enzymes with lysine-specific demethylase activity characterized [10]. LSD1 contains an amine oxidase domain, which demethylates proteins in a FAD-dependent manner, and a Swi3p, RSC8p, and Moira (SWIRM) domain, which is a characteristic of proteins that interact with chromatin. LSD1 exhibits enzymatic activity toward diand monomethyl histone $\mathrm{H} 3$ lysine 4 and lysine 9 (H3K4 and $\mathrm{H} 3 \mathrm{~K} 9$, respectively); the specificity for $\mathrm{H} 3 \mathrm{~K} 9$ arises when LSD1 binds to the androgen receptor (AR) resulting in a shift of its activity from H3K4 [11]. This highlights the key role of the LSD1-binding partners in determining its enzymatic targets. The demethylation of $\mathrm{H} 3 \mathrm{~K} 4$ results in repression of transcriptional activity, while the opposite occurs when H3K9 is demethylated, indicating a context-dependent effect of LSD1 on gene expression. This switch in specificity is aided by phosphorylation of threonine 6 of $\mathrm{H} 3$ by protein kinase $\mathrm{C} \beta 1$, which interacts with the LSD1-androgen receptor complex [12]. Several other LSD1-interacting partners have been identified, including the CoREST, CtBP, NRD, and BRAF35 complexes as well as Blimp-1, ZNF217 and ZNF198 [13-16]. The interaction of the LSD1/CoREST/ HDAC complex with SUMO-2 is important for the specific gene repression [17]. Similarly, Myc recruits LSD1 to specific chromatin regions, where it is required for efficient Myc-induced transcription [18]. These interac- tions occur primarily through the LSD1 tower domain, an insertion in the amine oxidase domain that extends as much as $90 \AA$ from the center of the protein.

The activity of LSD1 is not solely directed toward histone proteins. For example, LSD1 demethylates p53 when it is dimethylated at K370 [19]. This results in a loss of p53-53BP1 interaction, leading to a decrease in the promotion of apoptosis by $\mathrm{p} 53$, possibly contributing to cancer progression. p53 directly interacts with LSD1, and this interaction serves to promote LSD1 binding to specific promoters and its activity [20]. Demethylation of E2F1 by LSD1 promotes apoptosis by stabilizing the protein, allowing its accumulation through a mechanism involving the inhibition of the ubiquitination of the E2F1 protein [21]. Loss of $L S D 1$ in mouse embryonic stem cells results in a decrease in Dnmt1 protein levels [22], as methylation of Dnmt1 leads to its degradation. It is likely that further studies will identify other proteins that are the targets of LSD1 action.

We and others have generated LSD1-null mice and demonstrated that knockout embryos die during the early stages of development $[22,23]$. Further studies have begun to elucidate the role of LSD1 in various organ systems. Expression of $L S D 1$ is required for neural stem cell proliferation, and knockdown of $L S D 1$ in the brain results in decreased progenitor proliferation [24]. Interestingly, alternative splicing generates brain-specific LSD1 isoforms that affect neurite morphology [25], while in rats, upregulation of LSD1 expression occurs upon brain injury [26]. The interaction of LSD1 with TAL1, as part of a larger complex, is involved in maintaining erythroid cells in an undifferentiated state [27]. Recent work has also established that LSD1 is required for adipogenic differentiation, where its activity on methylated H3K9 primes the chromatin for upregulation of key factors involved in adipogenesis [28]. LSD1 also appears to play a role in human cancer, as it is overexpressed in tumors from several organs $[29,30]$. Conversely, examination of prostate cancer samples indicates minimal expression changes of LSD1 [31]. LSD1 expression is often decreased in human breast cancer, and ectopic expression of this protein is sufficient to decrease metastatic ability [32]. LSD1 interaction with Snail is required for repression of epithelial marker genes and to maintain the epithelial state in cancer cells $[33,34]$. In neuroblastomas, LSD1 expression is inversely correlated with the differentiation state of the cells, and knockdown of $L S D 1$ results in defects in cellular growth in xenograft models [35].

While many roles for LSD1 continue to be identified, its in vivo role beyond early development remains poorly characterized. This has been hampered in particular 
due to the fact that the LSD1-null mice die in utero. We sought to take advantage of the conditional floxed allele that was generated during the course of our previous study [22] to explore the function of LSD1 in specific tissues. However, the animals homozygous for the floxed allele exhibited a failure to survive after birth. This suggested that the floxed allele actually represented a hypomorphic allele, and would instead provide further insight into the role of LSD1 during the later stages of development. The hypomorphic animals suffered from heart defects, identified as the likely cause of perinatal lethality. The floxed allele turned out to contain two point mutations, which were subsequently shown to decrease both LSD1 enzymatic activity and binding to several known interactors. This resulted in the upregulation of a small subset of gene products, including the calcium/calmodulin kinase II (CK2) $\beta$ kinase subunit. In these hearts, Ecadherin, a target of the CK2 kinase, was hyperphosphorylated. These results thereby identify a novel pathway by which defects in LSD1 affect the proper development of the mammalian heart.

\section{Results}

\section{The floxed LSD1 allele is hypomorphic}

Previous reports $[22,23]$ demonstrated that embryos lacking a functional copy of $L S D 1$ die early during development, at approximately E6.5. We therefore attempted to examine the effect of LSD1 knockout in specific tissues by employing the Cre-lox technology. We sought to generate mice homozygous for the floxed allele $\left(A \circ f 2^{210 x / 210 x}\right)$. Unexpectedly, intercrosses of $A \circ 2^{210 x /+}$ mice resulted in no $A$ of $2^{2 \text { lox } / 210 x}$ animals reaching adulthood. A closer examination of the development of these mice indicated that at E13.5 and E18.5, the normal Mendelian ratio was present (Figure 1A); however, by 3 weeks of age no $A$ of $2^{2 \operatorname{lox} / 2 \operatorname{lox}}$ mice were present. This perinatal lethality is often a sign of a cardiovascular defect, where animals succumb following birth due to the defective circulation of oxygenated blood [1]

Phenotypic analysis of late-stage E18.5 embryos was accomplished by light microscopic evaluation of tissue sections in order to identify any potential developmental abnormalities. Of five E18.5 Aof $2^{2 \text { lox/2lox }}$ embryos that were completely serially sectioned and tissues of which were subsequently evaluated by light microscopy, three showed ventricular septal defects (VSDs) characterized by incomplete closure of the membranous portion of the ventricular septum (Figure 1B), and the other two showed a left atrio-ventricular valve defect and myodegeneration/mineralization of the heart, respectively. Similar VSDs were also identified in all four $A \circ f 2^{2 \text { lox/lox }}$ pups examined $(110 x=$ null allele $)$. For both $A o f 2^{210 x / 2 l o x}$ and $A$ of $2^{210 x / 10 x}$ pups that were examined by light microscopy, sectioned tissues from littermate wild-type pups served as appropriate controls. As no major defects in other organs and tissues were observed in $A o f 2^{2 \operatorname{lox} / 2 \operatorname{lox}}$ and Aof $2^{2 \text { lox/llox }}$ pups, we believe that the heart defects were primarily responsible for the perinatal lethal phenotype.

\section{The floxed allele contains two point mutations}

The loxP sites in the Aof $2^{2 l o x}$ allele are located in the introns of the gene [22], and are not supposed to affect

\begin{tabular}{|c|c|c|c|}
\hline \multicolumn{4}{|c|}{$2 \mathrm{lox} /+\mathrm{X} 2 \mathrm{lox} /+$} \\
\hline & E13.5 & E18.5 & P21 \\
\hline wt & 15.4 & 30.0 & 32.8 \\
\hline 2lox/wt & 46.2 & 36.7 & 67.2 \\
\hline 2lox/2lox & 38.5 & 33.3 & 0.0 \\
\hline
\end{tabular}

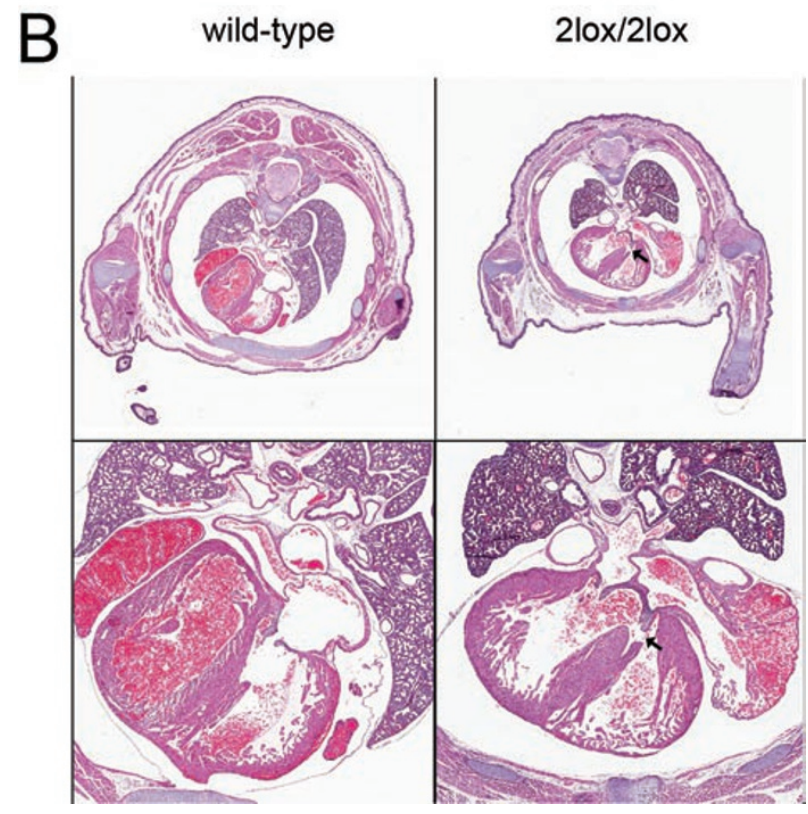

Figure 1 Mice homozygous for the floxed ("2lox") allele show heart defects. (A) Embryos homozygous for the 2lox allele survive to birth, but die shortly thereafter, as shown by the lack of homozygous pups at day p21. The data represents the percent of each genotype present at the indicated time point; for E13.5, this is the total of three litters, while E18.5 and p21 represent data from six pooled litters each. (B) Representative photomicrograph (hematoxylin and eosin staining) illustrating a ventricular septal defect in an E18.5 Aof2 ${ }^{2 \text { lox/1lox }}$ pup at low magnification (upper right hand panel; 1.0x) and higher magnification (lower right hand panel; $3.0 \times$ ) compared to a wild-type control animals (upper and lower left hand panels). 
protein function, so we examined whether the LSD1 coding sequence was altered during the generation of this allele. cDNAs from wild-type and $A o f 2^{2 \operatorname{lox} / 2 \operatorname{lox}}$ mouse embryonic fibroblasts were cloned and the coding sequences were compared by sequencing. The sequence of the 2 lox allele was found to contain two adenine-to-guanine point mutations, which had been accidentally introduced in the gene-targeting vector. The mutations resulted in two amino-acid changes (E413G and M448V) in the "tower" domain of LSD1, which is known to mediate proteinprotein interactions [36-38], and as such could have important negative effects on LSD1 function. As the crystal structure of LSD1 has been solved [36, 38, 39], the predicted effect of these point mutations on the structure and function of this protein was then modeled (Supplementary information, Figure S1). The amino acid at position 413 is located near the base of the tower domain, and is in close proximity to the catalytic domain. This could potentially affect the enzymatic activity of LSD1 and/or the structure of the tower domain. The mutation at 448 , conversely, occurs at a residue that plays a role in the binding between LSD1 and CoREST, a protein that is involved in modulating LSD1 activity [40], and so would be more likely to alter protein-protein interactions. Based on these models, it is therefore possible that Aof $2^{210 x / 210 x}$ mice are affected by decreased protein activity, altered protein-protein interactions, or both.

\section{The enzymatic activity of the 2loxLSD1 is altered}

In order to determine the effect of the point mutations on the enzymatic activity of LSD1, FLAG-tagged versions of the wild-type and 2loxLSD1 variants were generated, along with proteins containing each of the single point mutations present in the 2lox variant (E413G and M448V). This allowed for the dissection of the relative contribution of each point mutation to the defects in the LSD1 protein. In addition, a point mutant that is enzymatically dead (N535A [37]) was generated as a negative control. It is worth noting that we used a smaller LSD1 isoform (Genbank accession number BC059885), which lacks the $N$-terminal 50 amino acids of the full-length LSD1, as endogenous LSD1 from both embryonic hearts and MEF lines migrated at $\sim 90 \mathrm{kDa}$ (Supplementary information, Figure S2), which matched the predicted size of the short isoform and was smaller than the reported size of human LSD1 (although we cannot exclude the possibility that the size difference was simply due to the molecular size markers used in different laboratories). Furthermore, previous studies have shown that the unstructured $N$-terminal region of LSD1 is not required for enzymatic activity and does not seem to be involved in interacting with known binding partners [10, 38-42].
The FLAG-tagged proteins were expressed in HEK293T cells and purified using anti-FLAG resin. To eliminate the effects of interacting proteins on LSD1 activity, we used stringent conditions and purified the LSD1 proteins to near homogeneity (Figure 2A). The enzymatic activity of the 2lox variant was compared to the wild-type and enzymatically dead N535A LSD1, by incubating these proteins with purified bulk histone proteins and analyzing H3K4 methylation changes. Immunoblots performed following this incubation demonstrated a substantial decrease in the amount of H3K4me1 and $\mathrm{H} 3 \mathrm{~K} 4 \mathrm{me} 2$ present in the samples treated with wildtype LSD1 compared to the control N535A mutant. The 2lox variant, however, showed an intermediate activity, with a decrease in $\mathrm{H} 3 \mathrm{~K} 4 \mathrm{me} 1$ and $\mathrm{H} 3 \mathrm{~K} 4 \mathrm{me} 2$ compared to the N535A mutant but not to the same extent as the wild-type protein (Figure 2B). This is demonstrated graphically in Figure 2C, where quantification of a representative experiment demonstrates that the 2loxLSD1 has intermediate activity compared to the wild-type and enzymatically dead proteins.

In order to confirm these results, these purified LSD1 proteins were also tested for enzymatic activity toward a methylated peptide, using the production of hydrogen peroxide to examine enzyme function. The N535A mutant was indistinguishable from the control (no protein) sample, while the wild-type LSD1 showed significant demethylase activity (Figure 2D). Similar to the result with the purified histones, the 2loxLSD1 retained some activity but was not as active as the wild-type protein $(P<0.001)$. The E413G single mutant exhibited activity similar to the 2 lox protein. While the M $448 \mathrm{~V}$ protein demonstrated significantly higher activity than the 2 lox variant, its activity was less than that of the wild-type protein $(P<0.001$; Figure 2D). These results demonstrate that the 2loxLSD1 has reduced catalytic activity, primarily due to the mutation at amino acid 413 .

\section{The point mutations in 2loxLSD1 affect protein-protein interactions}

LSD1 interacts with numerous proteins, including CoREST, HDAC1, Zfp198, and the AR [13-16]. This could have important consequences for LSD1 function, as its binding partners determine the activity of LSD1. For example, LSD1 demethylates H3K9 only upon interaction with the androgen receptor [11]. Because the mutations in the 2loxLSD1 are located in the tower domain, the ability of LSD1 to bind to known partners was examined. Initially, complexes containing FLAG-tagged LSD1 variants were isolated from transiently transfected NIH 3 T3 cells, and the co-immunoprecipated proteins were identified by immunoblot. The wild-type LSD1 

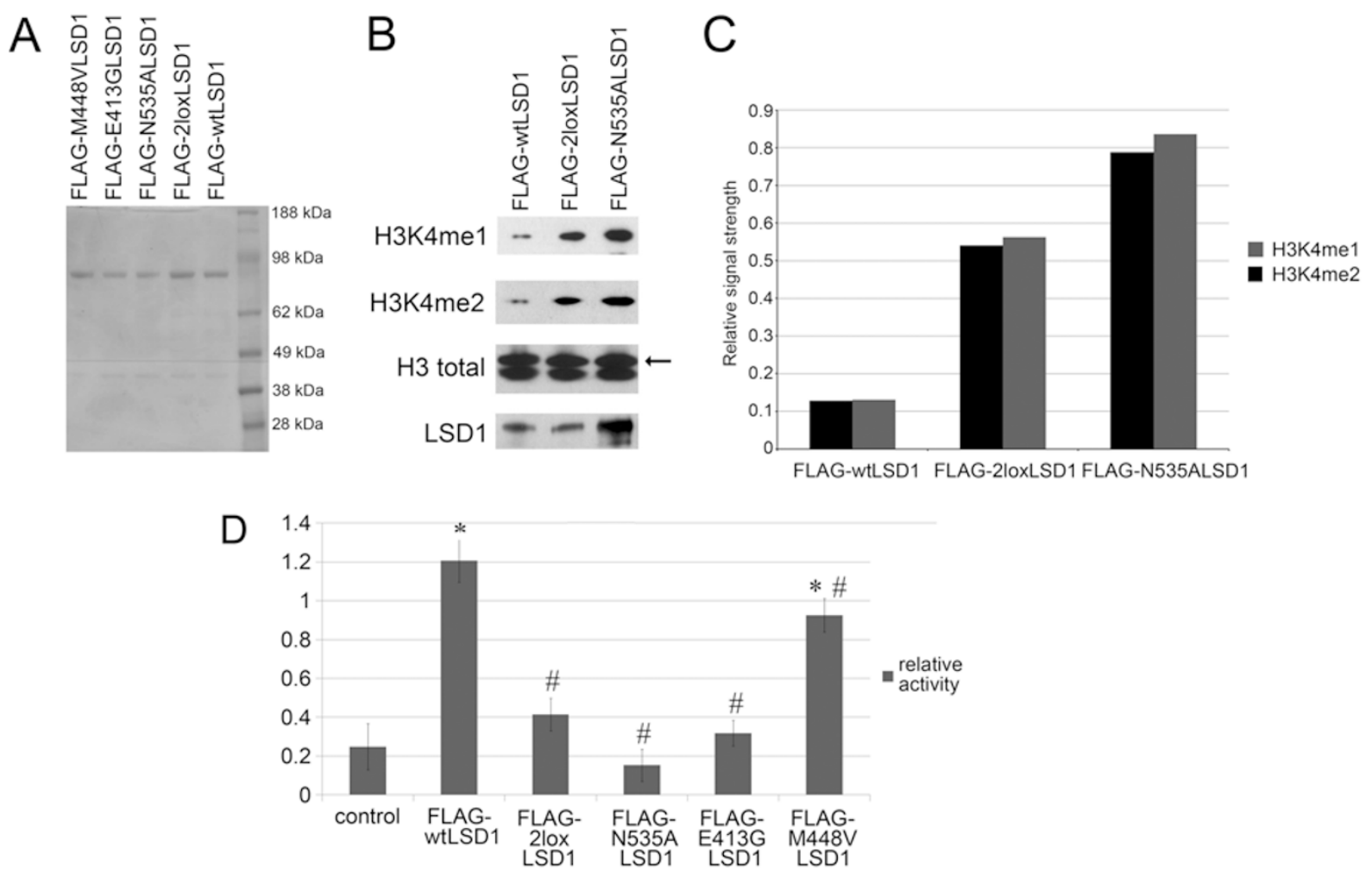

Figure 2 The enzymatic activity of the hypomorphic LSD1 is reduced. (A) FLAG-based isolation of LSD1 variants expressed in HEK293T cells. Coomassie blue staining demonstrates the purification of comparable amounts of the LSD1 variants. (B) The enzymatic activity of LSD1 was tested by incubating the purified proteins with bulk histones, and then analyzing by immunoblot. The 2lox variant of LSD1 shows intermediate activity, based on the reduction of H3K4me2 and H3K4me1, compared to the wild-type protein and the enzymatically-dead N535A mutant. (C) Quantitation of the reduction in H3K4me1 and $\mathrm{H} 3 \mathrm{~K} 4 \mathrm{me}$ 2, from a representative immunoblot following the treatment of bulk histones with the indicated purified FLAG-tagged proteins. Wild-type LSD1 shows a substantial reduction in both histone marks compared to the enzymatic-dead (N535A) mutant; the 2lox variant exhibits intermediate activity. Quantitation was performed with ImageJ software, using films with signal in the linear range, and normalized to total histone levels. (D) In vitro enzymatic activity demonstrates that the 2 lox variant and the E413G single mutant LSD1 show minimal activity that is only slightly greater than the no protein control or the N535A mutant. Conversely, the M448V mutant shows activity comparable to the wild-type LSD1. The data represents the mean \pm SD from three independent experiments. $* P<0.001$ compared to 2 loxLSD1; \# $P<0.001$ compared to wtLSD1.

protein interacted with CoREST and HDAC1, as did the N535A mutant, which demonstrated that the enzymatic activity of LSD1 is not required for binding (Figure 3A). On the other hand, the 2lox variant of LSD1 showed little-to-no binding to either of these proteins, indicating that the two mutations are sufficient to substantially alter the association of LSD1 with its interacting proteins. This was further confirmed by quantifying the binding of LSD1 to CoREST and HDAC1 (Figure 3B). The 2lox variant showed a significantly decreased ability to interact with both CoREST and HDAC1, compared to both the wild-type and N535A proteins. Examination of the single point mutants demonstrated that the M448V mutation was predominantly involved in the decrease in CoREST binding, as predicted by the structural model- ing (Supplementary information, Figure S1); the E413G mutant bound significantly more CoREST than the 2lox variant, but also significantly less than the wild-type protein.

To confirm the results in a more physiologically relevant system, the interaction between endogenous LSD1 and CoREST in primary mouse embryonic fibroblasts (MEFs) was examined. Significantly more LSD1 was pulled down by immunoprecipitation of CoREST in the wild-type MEFs compared to the 2lox/2lox MEFs. Similarly, substantially less CoREST was co-immunoprecipitated with the 2loxLSD1 compared to the wild-type protein (Figure 3C). Quantification of these results (Figure 3D) confirmed the significant difference in the interaction of LSD1 with CoREST in these cells. These results 

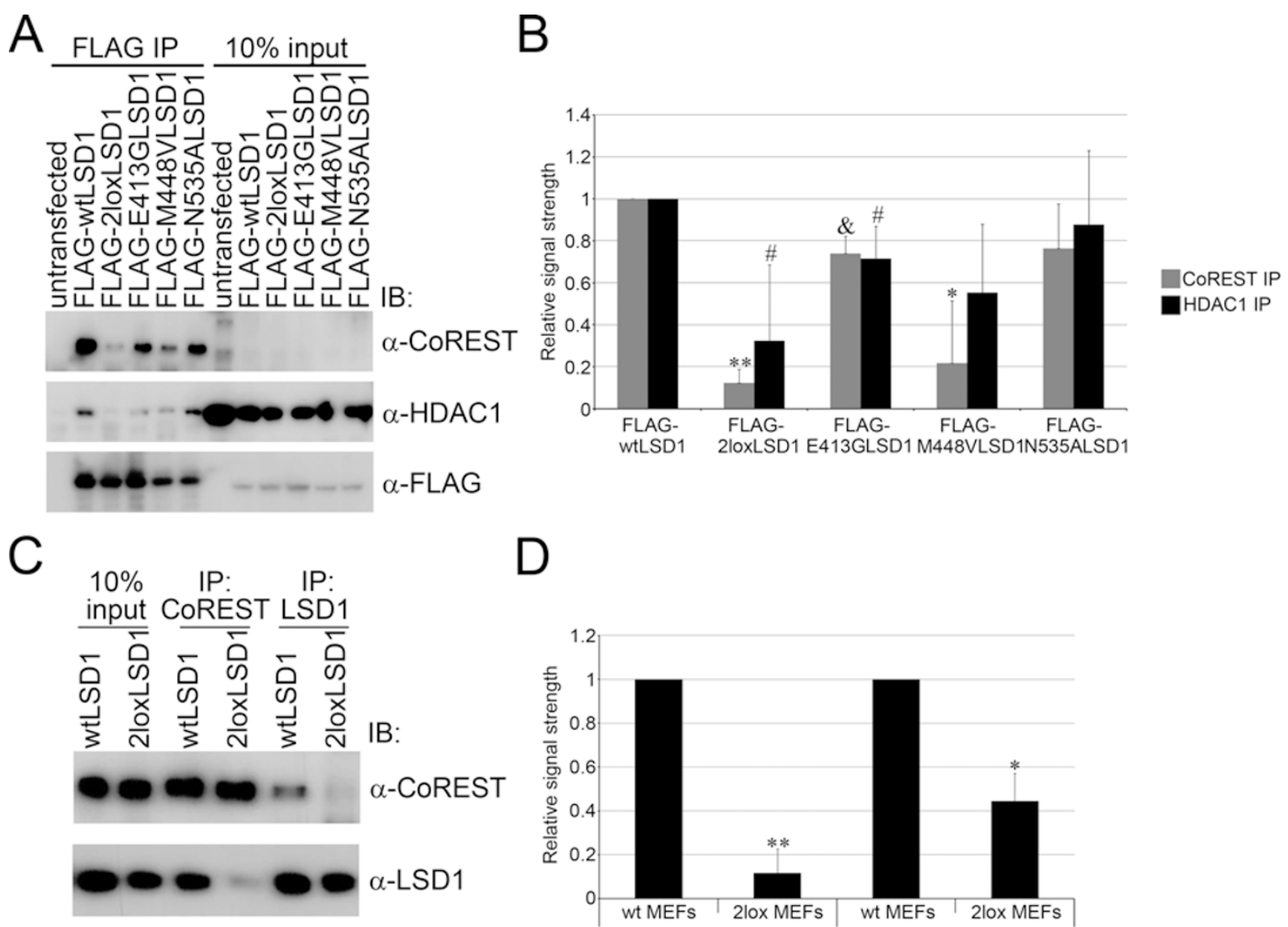

D

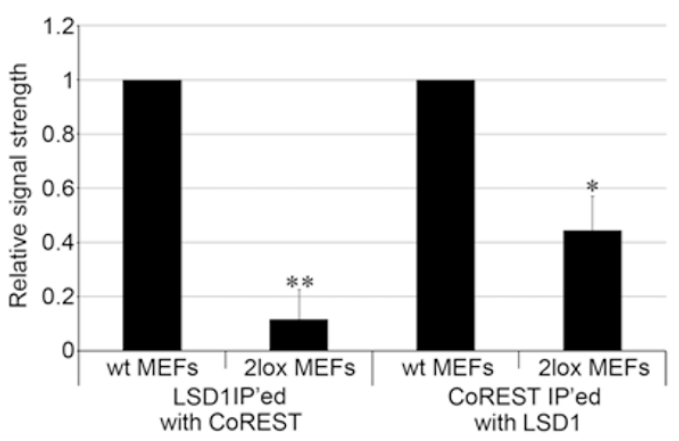

Figure 3 The 2lox LSD1 variant shows decreased binding to known interactors. (A) Immunoblots of FLAG-based coimmunoprecipitations of NIH 3 T3 cells transfected with the LSD1 variants demonstrate that the 2lox protein exhibits greatly reduced complex formation with known binding partners CoREST and HDAC1. The two single point mutants both exhibit intermediate binding to HDAC1, compared to the wild-type and 2lox proteins, while the M448V mutation has a more profound effect on CoREST binding. (B) The relative coimmunoprecipitation of the LSD1-binding partners CoREST and HDAC1 was quantitated using ImageJ software. Data represent the mean \pm SD for three independent experiments. ${ }^{* *} P<0.001 ; * P<0.005 ;{ }^{*} P<0.05$, all compared to wild-type LSD1 binding levels; \& $P<0.005$ compared to both wild-type LSD1 and 2lox LSD1. (C) Purification of endogenous LSD1 complexes from MEF lines confirmed a decrease in LSD1-CoREST interaction in cells expressing the mutant LSD1. All westerns are representative of results from experiments performed in triplicate. (D) The decrease in the endogenous interaction of LSD1 and CoREST by the 2lox variant was quantitated, using ImageJ software. Data represent the mean \pm SD for three independent experiments. ${ }^{* *} P<0.001$; $P<0.005$, compared to binding by wild-type LSD1.

indicate that the ability of the 2loxLSD1 variant to interact with its binding partners is compromised, which most likely affects its targeting and function.

\section{Hypomorphic hearts show no major cellular defects}

In order to determine the effect of the hypomorphic LSD1 allele on heart development at the cellular level, the hypomorphic and wild-type hearts were next compared for proliferation, apoptosis, and cellular migration. Pregnant females (E13.5) were injected intraperitoneally with BrdU, and the embryos were harvested $1 \mathrm{~h}$ later. The hearts from these embryos were then examined for proliferation and cardiomyocyte presence. BrdU incorporation in the wild-type and Aof $2^{2 l o x / 2 l o x}$ hearts was essentially identical (Supplementary information, Figure S3A), indicating that the cardiac defects were not the result of a proliferation defect. The defect in ventricular septation may also result from defective cardiomyocyte migration [43]. Therefore, the presence of cardiomyocytes in the developing septum of the embryos was examined to determine whether there was a defect in cellular population of this structure by staining for sarcomere myosin (Supplementary information, Figure S3B). However, no substantial difference in the cardiomyocyte presence was 
observed between the wild-type and hypomorphic hearts.

Increased E-cadherin phosphorylation in the hypomorphic hearts

In addition to microarray analysis of gene expression in the hypomorphic hearts (see following section), a candidate approach was employed to examine pathways involved in heart development. Lysates were extracted from E18.5 hearts and subjected to immunoblotting (Figure 4). LSD1 showed an approximately 50\% reduction in hypomorphic hearts, whereas $\mathrm{Nkx} 2.5$, an important transcription factor involved in heart development, was not altered. While the expression levels of Ncam and E-cadherin, adhesion molecules involved in heart development, did not appear to be substantially different between the wild-type and hypomorphic hearts, the phosphorylation of E-cadherin (on serines 838 and 840) was significantly increased in hypomorphic hearts. We also examined the levels of active and total $\beta$-catenin and observed no obvious differences between wild-type and 2lox/2lox hearts (Figure 4).

In order to confirm these results, we carried out immunohistochemistry on heart sections. In agreement with the immunoblotting data, there was a slight decrease in LSD1 staining (data not shown) and a general, major increase in the phosphorylated form of E-cadherin in the 2lox/2lox hearts compared to the wild-type hearts (Figure $5 \mathrm{~A})$. Phosphorylation of E-cadherin has been shown to enhance its affinity to bind $\beta$-catenin $[44,45]$. Indeed, the localization of $\beta$-catenin appeared altered in 2 lox/2lox hearts, with a greater proportion of the protein localized to the plasma membrane and a lower amount in the cytoplasm (Figure 5B). The levels and localization of other proteins examined, including Notch, total E-cadherin, Wnt, and VEGF, showed no obvious differences between wild-type and hypomorphic hearts (data not shown). Non-specific IgG staining was used as a negative control (Figure 5C). Together, our results suggest that LSD1 plays a role in controlling the balance of phosphorylation of E-cadherin in the heart.

\section{Upregulation of CK2 in the hypomorphic hearts}

Because LSD1 is known to modify gene expression [10, 11], microarrays were performed on RNA samples isolated from the hearts of wild-type and 2lox/2lox littermates at developmental day E18.5. The data from these microarrays were analyzed to identify gene products that showed significant expression changes between the hypomorphic and control hearts, with the cut off for significance being an adjusted $P$-value of 0.05 . Using these parameters, only 36 unique gene products showed significant changes between the control and the LSD1 hypomorphic hearts (Table

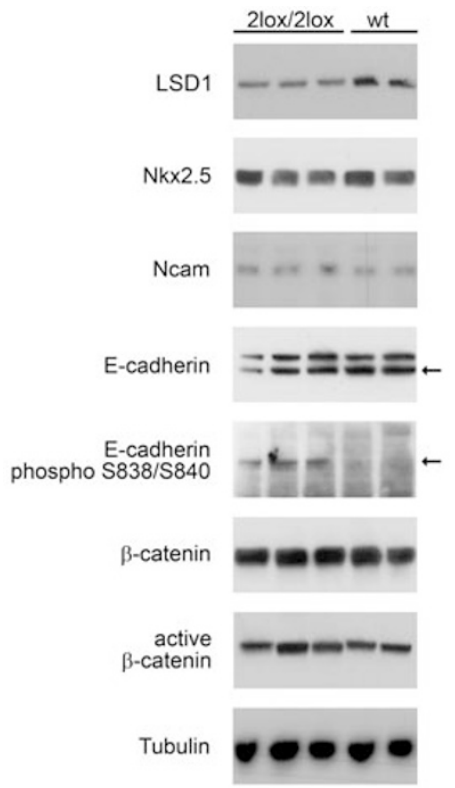

Figure 4 Protein expression in the developmentally defective hearts. The expression of proteins in three hypomorphic and two control hearts was examined by immunoblotting. A decrease in LSD1 and a major increase in phosphorylation of E-cadherin were observed in the Aof2 $2^{210 x / 210 x}$ hearts, whereas all other proteins examined showed no obvious changes. In the E-cadherin blot, the arrow indicates the correct E-cadherin band, and the identity of the higher band is unknown. The Ncam antibody recognizes the $140 \mathrm{kDa}$ isoform of this protein.

1 and Supplementary information, Table S1). Consistent with the role of LSD1 in transcriptional repression, the majority of the genes exhibiting alterations in their expression levels were upregulated in the Aof $2^{2 \operatorname{lox} / 2 \operatorname{lox}}$ hearts. Indeed, only six gene products were found to be downregulated, including $L S D 1$ (Aof2) itself. Intriguingly, the $\beta$ subunit of CK2, which is known to phosphorylate Ecadherin, was among the upregulated genes.

In order to confirm these results, RNA samples were isolated from hearts of E18.5 embryos for quantitative real-time polymerase chain reaction (qRT-PCR) analysis. As opposed to the wild-type controls, the hypomorphic hearts showed increased expression of $C K 2 \beta$, although the extent of the increase varied between 2 lox/2lox animals. The $\alpha$ subunit of CK2 (CK2 $\alpha)$ also showed a moderate increase. In contrast, the expression of glycogensynthase kinase $3 \beta$ (GSK3 $\beta$ ), which also phosphorylates E-cadherin [46], was slightly reduced in 2lox/2lox hearts (Figure 6A). These results suggest that the increase in CK2 expression may be responsible for the hyperphosphorylation of E-cadherin observed in hypomorphic hearts. Several other genes were also analyzed. The 
A
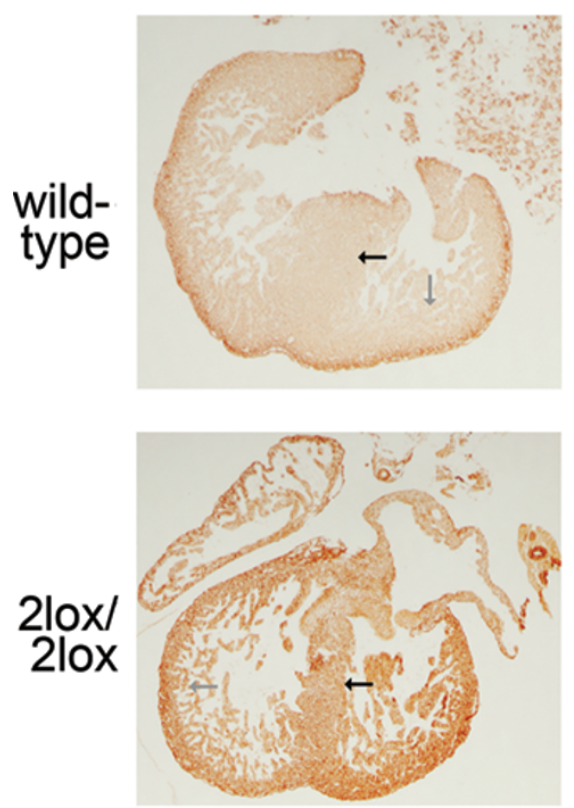

$40 x$, wall
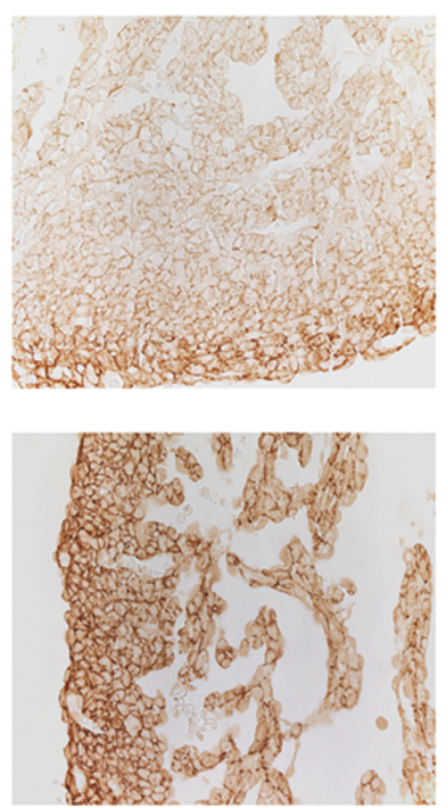

40x, septum
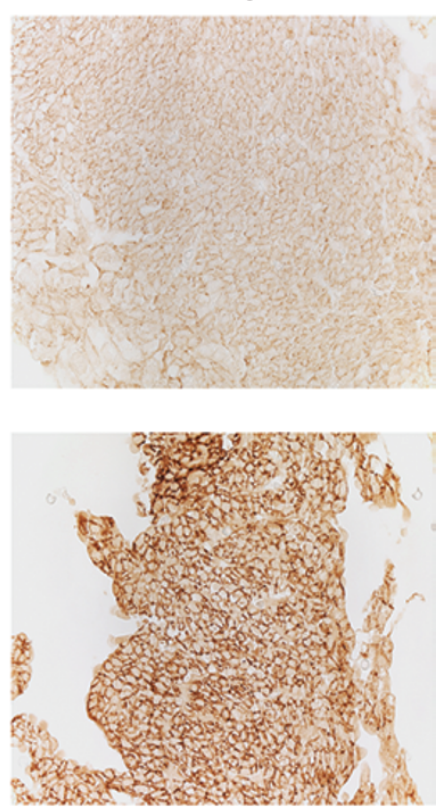

phospho-S838/S840 E-cadherin

B
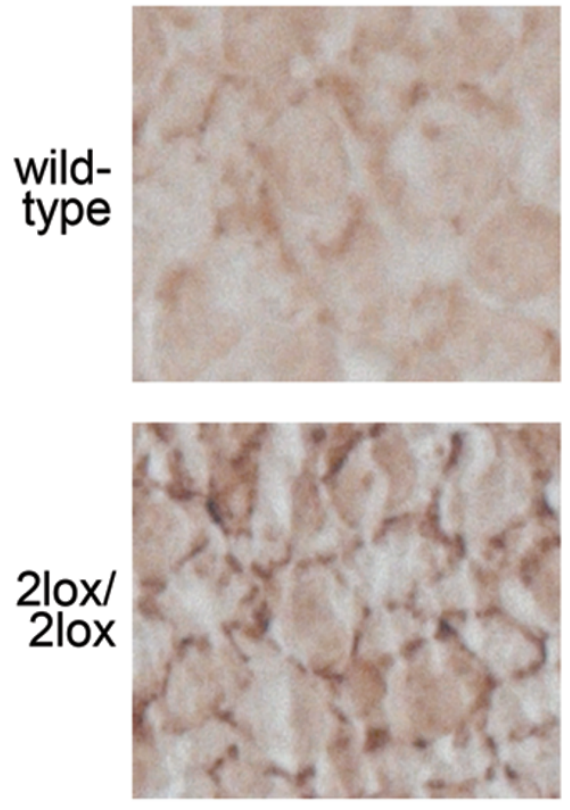

$\beta$-catenin
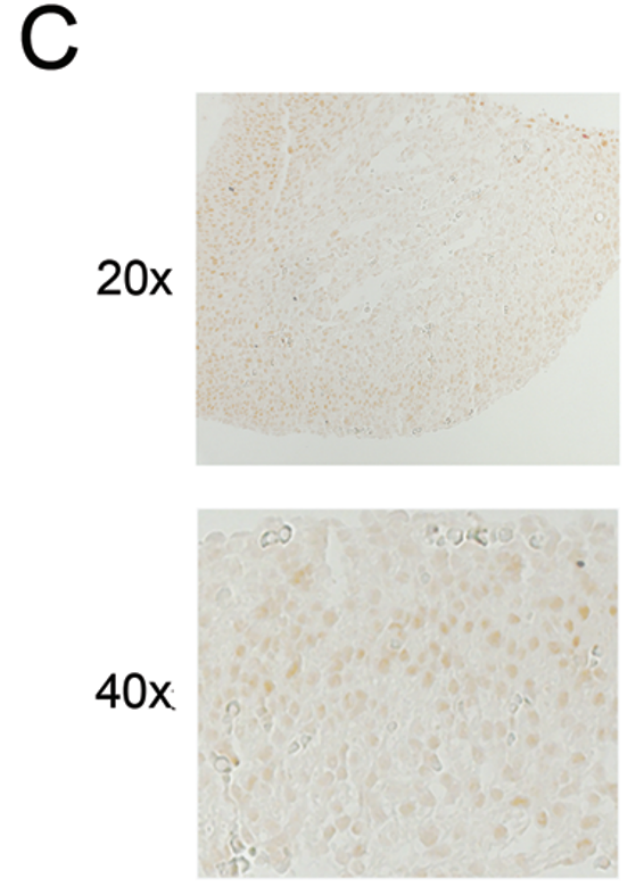

$\lg G$

Figure 5 Immunohistochemistry of the hypomorphic hearts. (A) Staining with an antibody specific for the phosphorylation of E-cadherin is strongly increased in the Aof $2^{2 \operatorname{lox} / 2 l o x}$ hearts compared to the control. The arrows indicate the regions (grey for heart wall, black for septum) from which the higher magnification images (on right) originate. (B) $\beta$-catenin localization is altered, with more of the protein present at the plasma membrane in Aof $2^{210 x / 2 l o x}$ hearts. (C) Staining with a non-specific IgG control antibody confirms the specificity of the staining. To minimize background, no counterstain was used. All photomicrographs constitute representative fields; magnification factor is provided above or beside the photographs. 
Table 1 Gene products that showed significantly changed expression in the hypomorphic hearts

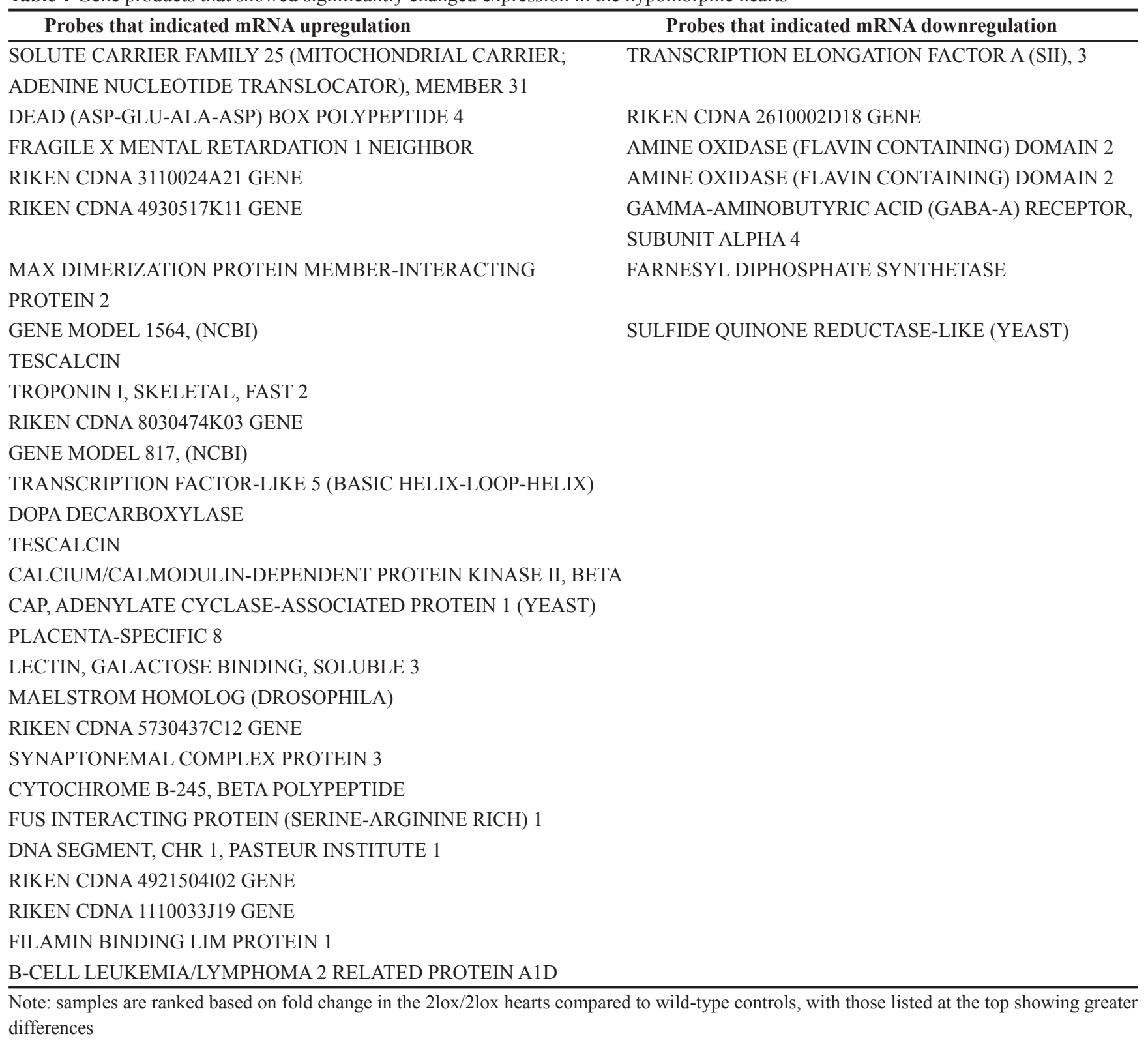

difference in mRNA levels of Fblim1, as observed by microarray, was not recapitulated in this case, while the increase in tescalcin was confirmed (Figure 6B). Tescalcin, which encodes an EF-hand calcium-binding protein, is highly expressed in the heart, but its role, if any, in heart development is largely unknown. Consistent with the immunoblotting and microarray data, $\mathrm{Nkx} 2.5$, $\beta$-catenin, and Ncam transcripts showed no alterations in the hypomorphic hearts, and mRNA levels of $L S D 1$ were decreased by approximately 50\% (Figure 6B). Aside from the two point mutations, no unintended genetic alterations were identified in the gene-targeting vector. The reduction of LSD1 transcript, therefore, could be due to the insertion of loxP sites, which might affect LSD1 transcription or processing. The reduction of LSD1 probably did not significantly contribute to the heart defects, as mice heterozygous for the LSD1 null allele (1lox/+), which produce similar amount of LSD1 as 2lox/2lox mice (Supplementary information, Figure S4), showed no obvious phenotype [22].

Despite the lack of Wnt pathway activation in the microarray results (Table 1), alterations in Wnt pathway targets were examined due to the increased phosphorylation of E-cadherin and the important role of this pathway dur- 
A

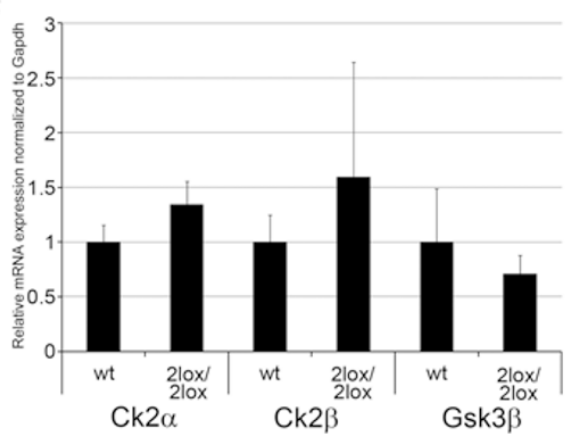

C

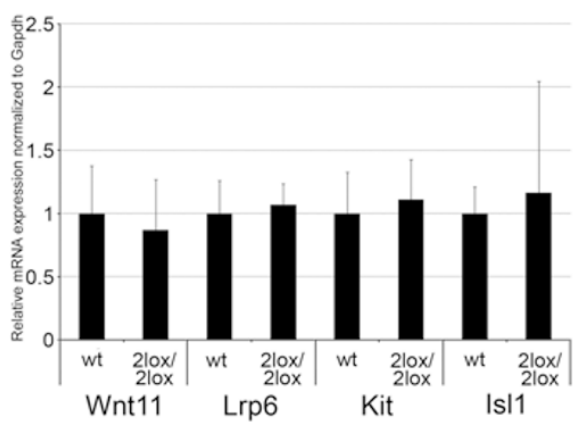

B

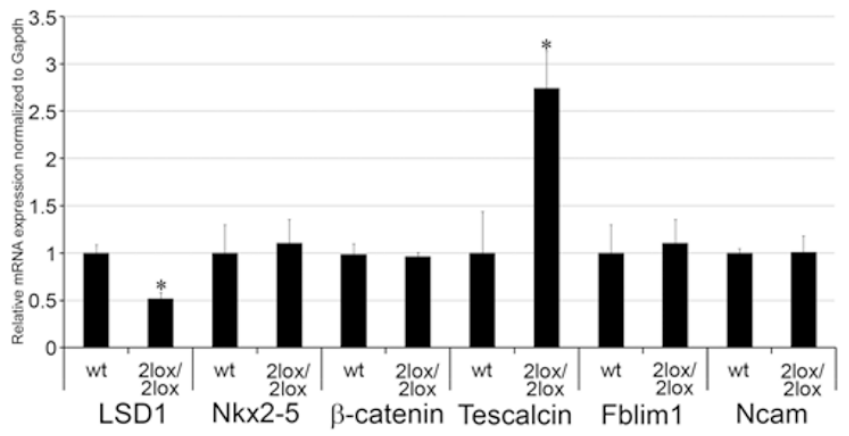

D

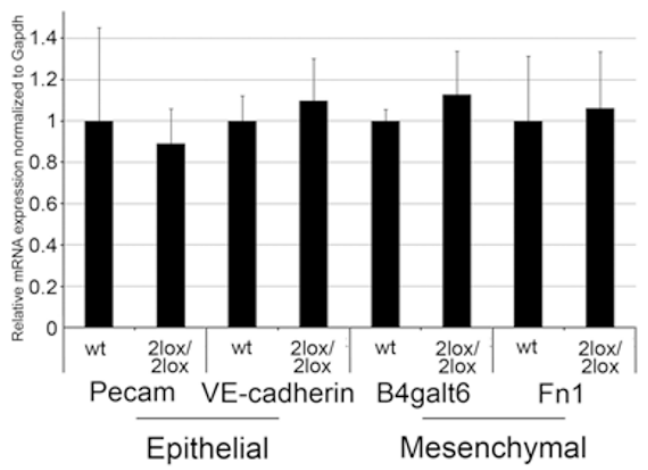

Figure 6 Changes in gene expression in the hypomorphic hearts. (A) Expression of subunits of CK2 and GSK3 $\beta$, examined by qRT-PCR using RNA isolated from E18.5 embryonic hearts. Data represents mean \pm SD from five animals, and was normalized such that the expression of the mRNA in wild-type animals was equal to 1. (B) Expression of other gene products in the hearts from the 2lox/2lox mice. LSD1 mRNA levels were decreased by approximately $50 \%$, confirming the microarray data. Nkx2-5, a heart marker, Ncam, and $\beta$-catenin were not found to be significantly altered in the hypomorphic hearts. Data represents the mean \pm SD for RNA expression from three wild type and three hypomorphic hearts. The expression of tescalcin and Fblim1, two proteins with potential roles in heart development that were identified by microarray, was also analyzed. The increase in tescalcin expression was recapitulated by the qRT-PCR, while no difference in Fblim1 expression between the wild type and hypomorphic hearts was observed. (C) Expression of Wnt targets in the wild type and hypomorphic hearts. The Wnt signaling pathway target genes Wnt11, Lrp6, Kit, and Isl1 were examined for changes in mRNA levels in the hypomorphic hearts by qRT-PCR. No statistically significant changes were observed in the expression of any of these genes. (D) mRNA expression of epithelial and mesenchymal markers is identical between wild-type and 2lox/2lox hearts. Expression of the epithelial markers Pecam and VE-Cadherin, and the mesenchymal markers B4galt6 and Fn1 was examined by qRTPCR using RNA isolated from E18.5 embryonic hearts. No statistically significant difference was noted in the expression of any of these mRNAs.

ing cardiac development [47]. Thus, the mRNA expression of Wnt11, low-density lipoprotein receptor-related protein 6 (Lrp6), Kit, and Islet1 (Is11), all of which are known to be targets of the Wnt pathway, was compared between the wild-type and hypomorphic animals. No major difference was observed in the expression of any of the genes (Figure 6C), confirming the microarray results. These results suggest that hyperphosphorylation of E-cadherin, despite its effect on $\beta$-catenin localization, had no major effect on the Wnt signaling pathway. One possibility is that the decrease in GSK3 $\beta$, a negative regulator of the Wnt pathway, had a compensatory effect.
LSD1 interacts with Snail, which plays a role in the regulation of epithelial-mesenchymal transition (EMT) [33]. Because the formation of a complete ventricular septum requires, in part, that cells undergo an EMT, we examined the expression of epithelial and mesenchymal markers in the hypomorphic hearts. The expression of Pecam1 and VE-cadherin, two epithelial markers, and of UDP-Gal: $\beta$ GlcNAc $\beta$ 1,4-galactosyltransferase, polypeptide 6 (B4galt6) and Fibronectin1 (Fn1), two mesenchymal markers, was thus determined by qRT-PCR. No significant change in expression was observed for any of these RNAs (Figure 6D), suggesting that EMT was not 
affected in the defective hearts.

\section{Discussion}

In this study, we have identified a previously unknown role for the lysine demethylase LSD1 in cardiac development in mice. Previous reports examining LSD1 function in vivo employed knockout mice that showed early embryonic lethality (at approximately E6.5) [22, 23], thereby precluding an examination of the contribution of LSD1 to later stages of development. We have characterized mice homozygous for an Aof 2 allele that encodes a hypomorphic protein containing two point mutations in the tower domain. This LSD1 variant exhibits reduced enzymatic activity (Figure 2) as well as decreased binding to known interacting partners (Figure 3), although it does retain residual amounts of both activities. The resulting mice demonstrated cardiac development defects, primarily in the form of VSDs (Figure 1). This study therefore establishes a role for LSD1 during this developmental process.

Expression analysis of the hypomorphic $L S D 1$ hearts demonstrated that only a small subset of gene products showed altered expression in these hearts (Table 1). While LSD1 has been shown to play a role in both activation and repression of specific genes, depending on the context [23], overexpression of this protein does not result in an appreciable global decrease in $\mathrm{H} 3 \mathrm{~K} 4$ methylation (data not shown), in contrast to the closely related AOF1/LSD2/KDM1B [48]. This would appear to indicate that LSD1 activity is selective for specific promoters and may explain the minor alterations of gene expression. None of the genes identified by microarray are known to be critically involved in heart development, although reports have indirectly implicated some of the proteins. For example, tescalcin is expressed in the developing heart $[49,50]$ and modulates the function of calcineurin and the $\mathrm{Na}^{+} / \mathrm{H}^{+}$exchanger Nhe1 [50-52]. Notably, tescalcin interacts with Nhe1 and inhibits the activity of Nhe1 [51], a protein that plays an important role in the differentiation of cardiomyocytes [53]. It is therefore tempting to postulate that the defect arises due to the overexpression of tescalcin. However, inhibition of Nhe1 results in decreased levels of several important cardiac transcription factors, including Nkx2-5 [53]. Our results demonstrated no alteration in the expression of $\mathrm{Nkx} 2-5$ at either the mRNA or protein levels (Figures 4 and 6B), arguing against altered Nhe1 activity in the hypomorphic hearts. It is possible, however, that tescalcin acts in an Nhe1-independent pathway. Further work is required to determine the significance of tescalcin upregulation in the heart defects observed in the LSDI hypomorphic mice.

Because of the lack of promising proteins identified by microarray, we undertook a candidate approach, whereby pathways known to be involved in cardiac development were analyzed by immunoblotting and immunohistochemistry for alterations in the hypomorphic hearts. Among the molecules examined was E-cadherin, which not only has a role in heart development [54], but also is a target of LSD1-mediated transcriptional repression [33]. Total E-cadherin levels were essentially unchanged in the heart, but a major increase in phosphorylated E-cadherin was observed (Figures 4 and 5), suggesting that while the overall levels of this protein are not altered, its functional state is different.

Cell adhesion is closely regulated in the heart [55]. For example, Ncam is expressed in a specific manner during chick heart development [56]. Expression of the tenascin variants TNC and TNX, which are important for tissue remodeling, also varies greatly during heart development [57]. N-cadherin is essential for embryonic development, with knockout animals showing heart defects that can be partially overcome by reexpressing this protein specifically in muscles [58]. The altered phosphorylation status of E-cadherin in the hearts may affect cell-cell adhesion in this organ. Phosphorylation of E-cadherin results in significantly enhanced binding to $\beta$-catenin, by as much as 300 -fold $[44,45]$. In breast cancer cells, phosphorylation of $\beta$-catenin by the kinase CKI $\alpha$ results in the increased formation of E-cadherin $/ \beta$-catenin complexes and increases intercellular adhesion [59]. Thus, the increased phosphorylation of E-cadherin in the LSD1 hypomorphic hearts likely results in strengthened intercellular adhesion. Immunohistochemistry analysis of the hypomorphic hearts demonstrated an enrichment of $\beta$-catenin at the plasma membrane (Figure 5B), consistent with this model. Phosphorylation of E-cadherin is mediated in vitro by both GSK3 $\beta$ and CK2 $[45,46]$. Our data demonstrated that both the $\alpha$ and $\beta$ subunits of the CK2 kinase were upregulated in the hearts of the hypomorphic animals, while GSK3 $\beta$ levels showed a mild decrease. CK2 $\beta$-null embryos die early during development, with resorption occurring by day E7.5 [60]. Heterozygous-null embryos containing one knockout allele show reduced survival, suggesting that the dosage of CK2 $\beta$ in the embryo is important for proper development [61]. Similarly, deletion of the $\alpha$ subunit of CK2 results in mid-gestational lethality with embryos demonstrating several defects, including in the heart development [62]. CK2-mediated phosphorylation of E-cadherin affects both localization and function of this adhesion molecule [63], ultimately leading to an increase in its adhesive properties [46]. Although we did not notice a significant 
change in cardiomyocyte migration into the septum (Supplementary information, Figure S3), it is possible that the increased adhesiveness of the phosphorylated E-cadherin interferes with the final closure of the septum of the heart in the hypomorphic animals. Future studies to confirm the role of $C K 2 \beta$ in the cardiac developmental phenotype observed may employ mice heterozygous for the CK2 $\beta$ knockout allele [61] in combination with the mutant LSD1 allele. Reversal of the increased phosphorylation of E-cadherin and of the VSDs by decreasing the levels of CK2 $\beta$ would confirm the role of this kinase subunit in the developmental defect.

Other possible mechanisms by which the LSD1 hypomorphic allele leads to cardiac defects can be envisaged. For example, alterations in EMT may underlie some of the defects, including VSDs, given that LSD1 plays an important role in modulation of EMT [33] and that the development of the ventricular septum and heart valves relies on EMT of endocardial cells. However, several lines of evidence argue against a major defect in EMT in LSD1 hypomorphic animals. First, 2lox/2lox pups were able to develop to term without major defects in other organs and tissues, suggesting that pathways involved in EMT were largely unperturbed. Second, examination of the expression of epithelial and mesenchymal markers in the hypomorphic hearts demonstrated no appreciable difference compared to wild-type hearts (Figure 6D). Finally, E-cadherin protein level was not altered in the hypomorphic hearts (Figure 4), indicating that the repressive activity of Snail on E-cadherin, which requires LSD1, was not affected. Nevertheless, we cannot exclude the possibility that subtle changes in EMT may have contributed to the heart defects observed in LSDI hypomorphic mice.

Defects in cardiac development due to changes in gene dosage are becoming a common observation. For example, insufficiency in the $T A B 2$ gene results in congenital heart defects in human and zebrafish [64]. In mice, the loss of a single allele of the critical cardiac transcription factor Nkx2-5 results in heart developmental defects [65]. Tbx 5 insufficiency alters the expression of genes critical for cardiac development and leads to proliferation and migration defects [66]. The cardiac transcription factors Hand1 and Hand 2 also show gene dosage effects on cardiac development, with decreased expression leading notably to ventricular abnormalities [67]. Because the expression of developmental genes must be maintained in the correct spatio-temporal pattern, it would seem likely that epigenetic factors will play an important role in regulating this process. Indeed, the role of epigenetic factors in heart development has gradually been coming to light. BAF60C, a member of the BAF chromatin- remodeling complex, serves as a bridge linking cardiac transcription factors, including Gata4, to this complex [68]. A partial loss of BAF60C is sufficient to affect outflow tract development; complete knockout results in even greater defects. Similarly, the deletion of the lysine methyltransferase BOP results in defects in cardiomyocyte differentiation and development of the right ventricle [69]. Cardiac-specific disruption of the H3K79 methyltransferase Dot1L results in chamber dilation, increased cardiomyocyte cell death, systolic dysfunction, and conduction abnormalities [20]. Whole-body deletion of HDAC2 results in perinatal lethality due to a range of heart defects, while heart-specific deletion of HDAC1 and HDAC2 also results in malformed hearts and death at birth [70]. This study provides the first evidence that LSD1 plays a key role in the final stages of heart development in the mammalian embryo.

In summary, we have identified a role for the lysine demethylase LSD1 in the development of the mammalian heart, likely through effects on the phosphorylation of E-cadherin. Mice containing a hypomorphic variant of LSD1 demonstrate a highly penetrant defect in the formation of the septum separating the ventricles. However, there are only a small number of genes that are misregulated in the heart. Hyperphosphorylation of E-cadherin, probably due to upregulation of CK2 subunits, may be sufficient to interfere with migration of the cells, leading to the formation of VSDs. VSDs are common congenital defects in human infants, and future work examining the status of LSD1 in children with heart developmental defects will clarify the role of this protein in human cardiac malformation.

\section{Materials and Methods}

\section{Animals}

The mice used in these experiments, and the generation of the Aof 2 floxed allele, have been previously described [22]. All animal procedures employed in this study were approved by the Novartis Institutes for BioMedical Research Institutional Animal Care and Use Committee.

\section{Antibodies}

Antibodies used in this study were: rabbit anti-LSD1 (Cell Signaling, 2139), mouse anti-tubulin (Calbiochem, CP06), mouse anti-FLAG M2 (Sigma, F 1804), mouse anti-active- $\beta$-catenin (Millipore, 05-665), rabbit anti-phosphoS838+S840 E-cadherin (Abcam, ab76319), rabbit anti E-cadherin (Abcam, ab53033), mouse antisarcomere myosin (Developmental Studies Hybridoma Bank, University of Iowa, MF20), rabbit anti-HDAC1 (Abcam, ab19845), rabbit anti-CoREST (Abcam, ab32631), rabbit anti- $\beta$-catenin (Abcam, ab6302), rabbit anti-NCAM (Cell Signaling Technology \#3606), rabbit anti-monomethyl-Histone H3(Lys4) (Millipore, 07436), rabbit anti-dimethyl-Histone H3(Lys4) (Millipore, 07-030), 
and rabbit anti-Histone H3 (Millipore, 07-690). Immunoblotting and immunohistochemistry procedures were carried out employing standard protocols and antibodies at the manufacturer's recommended dilutions; chemiluminescence of immunoblots was developed using ECL-plus (GE Life Sciences). Protein molecular weight markers were purchased from Invitrogen (Cat\# LC5925).

\section{Histopathology}

To morphologically phenotype hypomorphic animals by light microscopy, embryos at known developmental stages from Aof $2^{2 l o x /+}$ intercrosses were dissected out of deciduas and fixed in $10 \%$ neutral buffered formalin for $24 \mathrm{~h}$. Samples were subsequently routinely processed, embedded in paraffin, and serially sectioned at $5.0 \mu \mathrm{m}$. Tissue sections were routinely stained with hematoxylin and eosin, and then examined by bright field light microscopy by a board certified veterinary pathologist for any potential morphological abnormalities.

\section{LSD1 cloning and mutagenesis}

LSD1 wild-type and 2lox cDNA was generated by isolating RNA from primary mouse embryonic fibroblast (MEF) cell lines homozygous for the respective $L S D 1$ allele. Total cellular RNA was converted to cDNA using SuperScript reverse transcriptase (Invitrogen) and an oligo dT primer, and then the LSD1 sequence amplified using high-fidelity KOD polymerase (Novagen) and specific primers (see Supplementary information, Table S2). The amplified cDNA was cloned into the p3XFLAG-myc-CMV-26 vector (Sigma-Aldrich) (the C-terminal myc tag would not be expressed because of the presence of a stop codon in the LSD1 cDNA). These constructs were sequenced on both the template and complementary strands, in duplicate, to identify point mutations in the 2lox coding sequence. Site-directed mutagenesis to generate single point mutants employed the primers described in Supplementary information, Table S2 and the QuikChange Site-Directed Mutagenesis Kit (Stratagene). All constructs were subsequently sequenced to verify mutagenesis.

\section{Immunoprecipitations}

NIH 3T3 cells were transiently transfected using Lipofectamine 2000 (Invitrogen) with vectors expressing FLAG-tagged LSD1 variants. $48 \mathrm{~h}$ later, the cells were lysed with FLAG complex buffer (10mM Tris, $\mathrm{pH} 8.0,140 \mathrm{NaCl}, 1.5 \mathrm{mM} \mathrm{MgCl}_{2}, 1 \mathrm{mM}$ DTT, 0.5\% Nonidet P-40, $0.1 \mathrm{mM} \mathrm{Na} \mathrm{VO}_{4}, 10 \mathrm{mM} \mathrm{NaF}, 10 \mathrm{mM}$ glycerol phosphate, and $1 \times$ protease inhibitor cocktail), and the lysate incubated with anti-FLAG resin (Sigma-Aldrich) for $3 \mathrm{~h}$ at $4{ }^{\circ} \mathrm{C}$. The beads were subsequently washed with $4 \times 1$ lysis buffer, and then resuspended in reducing SDS-PAGE (sodium dodecyl sulphate-polyacrylamide agarose gel electrophoresis) sample buffer and analyzed by immunoblot. For immunoprecipitation of endogenous complexes, 90\% confluent MEF lines were lysed with the same lysis buffer, and pre-cleared with Protein A/G Plus beads (Santa Cruz Biotechnology) for $1 \mathrm{~h}$ at $4{ }^{\circ} \mathrm{C}$. Samples were then left overnight with primary antibody (1:100 dilution). Protein A/ $\mathrm{G}$ Plus beads were added to the samples, which were left shaking at $4 \mathrm{C}$ for $2 \mathrm{~h}$. The beads were then washed with $4 \times$ lysis buffer and resuspended in SDS-PAGE sample buffer for immunoblotting. For changes in immunoblot intensity, films containing bands in the linear exposure range were scanned and analyzed by the ImageJ program (http://www.rsbweb.nih.gov/ij) for relative density mea- surements. Statistical significance was determined using the $t$-test (http://www.physics.csbsju.edu/stats/t-test_bulk_form.html).

\section{In vitro demethylase assays}

Demethylase assay with immunoblot was performed as described previously [48]. Briefly, vectors encoding FLAG-tagged LSD1 variants were transiently transfected into HEK293T using Lipofectamine 2000, and left for $48 \mathrm{~h}$ to allow for protein expression. Cells were lysed, and the LSD1 proteins purified using an anti-FLAG affinity column. These purified proteins were incubated with bulk histones (Sigma-Aldrich), at a 1:5 w/w ratio, for $4 \mathrm{~h}$ at $30{ }^{\circ} \mathrm{C}$. The lysine modification of the histones was then analyzed by immunoblot. In addition, the purified LSD1 proteins were tested for enzymatic activity in a fluorescence-based assay (BPS Bioscience), employing the manufacturer's protocol.

\section{RNA expression analysis}

Total RNA samples were isolated from freshly dissected E18.5 hearts using an RNeasy Plus kit (QIAGEN). RNA was then hybridized to Affymetrix Mouse430 GeneChip. Analysis of the results from the microarrays was performed as previously described [71]. Briefly, statistical significance was determined using false discovery rate corrected $P$-values, calculated using a Benjamini and Hochberg correction. Corrected $P$-values of 0.05 were used as the cutoff for significance, corresponding to a significance threshold of $\mid$ fold change $\mid>1.4$, adjusted $P$-value $<0.05$, using a moderated $t$-statistic (LIMMA).

For qRT-PCR analysis, $1 \mu \mathrm{g}$ of total RNA was reverse transcribed using iScript (BioRad) according to the manufacturer's instructions. qPCR reactions were then performed using TaqMan Gene Expression Assays (Applied Biosystems; for primers used see Supplementary information, Table S3) and an ABI7500 Fast Real-Time PCR System. Relative mRNA levels were calculated through comparison with GAPDH amplification values.

\section{Acknowledgments}

We thank Jeyaseelan Raju for helpful discussion and comments. This work was funded by the Novartis Institutes for BioMedical Research.

\section{References}

1 Conway SJ, Kruzynska-Frejtag A, Kneer PL, Machnicki M, Koushik SV. What cardiovascular defect does my prenatal mouse mutant have, and why? Genesis 2003; 35:1-21.

2 Bruneau BG. Mouse models of cardiac chamber formation and congenital heart disease. Trends in Genetics 2002; 18:S15-S20.

3 Savolainen SM, Foley JF, Elmore SA. Histology atlas of the developing mouse heart with emphasis on E11.5 to E18.5. Toxicol Pathol 2009; 37:395-414.

4 Buckingham M, Meilhac S, Zaffran S. Building the mammalian heart from two sources of myocardial cells. Nat Rev Genet 2005; 6:826-835.

5 Eisenberg LM, Markwald RR. Molecular regulation of atrioventricular valvuloseptal morphogenesis. Circ Res 1995; 77:1-6. 
6 Bentham J, Bhattacharya S. Genetic mechanisms controlling cardiovascular development. Ann N Y Acad Sci 2008; 1123:10-19.

7 Pierpont ME, Basson CT, Benson DW Jr, et al. Genetic basis for congenital heart defects: current knowledge: a scientific statement from the American Heart Association Congenital Cardiac Defects Committee, Council on Cardiovascular Disease in the Young: endorsed by the American Academy of Pediatrics. Circulation 2007; 115:3015-3038.

8 Kelly RG, Brown NA, Buckingham ME. The arterial pole of the mouse heart forms from Fgf10-expressing cells in pharyngeal mesoderm. Dev Cell 2001; 1:435-440.

9 Kwon C, Qian L, Cheng P, Nigam V, Arnold J, Srivastava D. A regulatory pathway involving Notch1/beta-catenin/Is11 determines cardiac progenitor cell fate. Nat Cell Biol 2009; 11:951-957.

10 Shi Y, Lan F, Matson C, et al. Histone demethylation mediated by the nuclear amine oxidase homolog LSD1. Cell 2004; 119:941-953.

11 Metzger E, Wissmann M, Yin N, et al. LSD1 demethylates repressive histone marks to promote androgen-receptordependent transcription. Nature 2005; 437:436-439.

12 Metzger E, Imhof A, Patel D, et al. Phosphorylation of histone H3T6 by PKCbeta(I) controls demethylation at histone H3K4. Nature 2010; 464:792-796.

13 Banck MS, Li S, Nishio H, Wang C, Beutler AS, Walsh MJ. The ZNF217 oncogene is a candidate organizer of repressive histone modifiers. Epigenetics 2009; 4:100-106.

14 Cowger JJ, Zhao Q, Isovic M, Torchia J. Biochemical characterization of the zinc-finger protein 217 transcriptional repressor complex: identification of a ZNF217 consensus recognition sequence. Oncogene 2007; 26:3378-3386.

15 Gocke CB, Yu H. ZNF198 stabilizes the LSD1-CoRESTHDAC1 complex on chromatin through its MYM-type zinc fingers. PLoS ONE 2008; 3:e3255.

16 Su ST, Ying HY, Chiu YK, Lin FR, Chen MY, Lin KI. Involvement of histone demethylase LSD-1 in Blimp-1-mediated gene repression during plasma cell differentiation. $\mathrm{Mol}$ Cell Biol 2009; 29:1421-31.

17 Ouyang J, Shi Y, Valin A, Xuan Y, Gill G. Direct binding of CoREST1 to SUMO-2/3 contributes to gene-specific repression by the LSD1/CoREST1/HDAC complex. Mol Cell 2009; 34:145-154.

18 Amente S, Bertoni A, Morano A, Lania L, Avvedimento EV, Majello B. LSD1-mediated demethylation of histone H3 lysine 4 triggers Myc-induced transcription. Oncogene 2010; 29:3691-3702.

19 Huang J, Sengupta R, Espejo AB, et al. p53 is regulated by the lysine demethylase LSD1. Nature 2007; 449:105-108.

20 Tsai WW, Nguyen TT, Shi Y, Barton MC. p53-targeted LSD1 functions in repression of chromatin structure and transcription in vivo. Mol Cell Biol 2008; 28:5139-5146.

21 Kontaki H, Talianidis I. Lysine methylation regulates E2F1induced cell death. Mol Cell 2010; 39:152-160.

22 Wang J, Hevi S, Kurash JK, et al. The lysine demethylase LSD1 (KDM1) is required for maintenance of global DNA methylation. Nat Genet 2009; 41:125-129.

23 Wang J, Scully K, Zhu X, et al. Opposing LSD1 complexes function in developmental gene activation and repression pro- grammes. Nature 2007; 446:882-887.

24 Sun G, Alzayady K, Stewart R, et al. Histone demethylase LSD1 regulates neural stem cell proliferation. Mol Cell Biol 2010; 30:1997-2005.

25 Zibetti C, Adamo A, Binda C, et al. Alternative splicing of the histone demethylase LSD1/KDM1 contributes to the modulation of neurite morphogenesis in the mammalian nervous system. J Neurosci 2010; 30:2521-2532.

26 Zhang YZ, Zhang QH, Ye H, et al. Distribution of lysinespecific demethylase 1 in the brain of rat and its response in transient global cerebral ischemia. Neurosci Res 2010; 68:6672.

$27 \mathrm{Hu} \mathrm{X}, \mathrm{Li}$ X, Valverde K, et al. LSD1-mediated epigenetic modification is required for TAL1 function and hematopoiesis. Proc Natl Acad Sci USA 2009; 106:10141-10146.

28 Musri MM, Carmona MC, Hanzu FA, Kaliman P, Gomis R, Parrizas M. Histone demethylase LSD1 regulates adipogenesis. J Biol Chem 2010; 285: 30034-41.

29 Hayami S, Kelly JD, Cho HS, et al. Overexpression of LSD1 contributes to human carcinogenesis through chromatin regulation in various cancers. Int J Cancer 2010; 128:574-86.

30 Lim S, Janzer A, Becker A, et al. Lysine-specific demethylase 1 (LSD1) is highly expressed in ER-negative breast cancers and a biomarker predicting aggressive biology. Carcinogenesis 2010; 31:512-520.

31 Suikki HE, Kujala PM, Tammela TL, van Weerden WM, Vessella RL, Visakorpi T. Genetic alterations and changes in expression of histone demethylases in prostate cancer. Prostate 2010; 70:889-898.

32 Wang Y, Zhang H, Chen Y, et al. LSD1 is a subunit of the NuRD complex and targets the metastasis programs in breast cancer. Cell 2009; 138:660-672.

33 Lin T, Ponn A, Hu X, Law BK, Lu J. Requirement of the histone demethylase LSD1 in Snail-mediated transcriptional repression during epithelial-mesenchymal transition. Oncogene 2010; 29:4896-904.

34 Lin Y, Wu Y, Li J, et al. The SNAG domain of Snaill functions as a molecular hook for recruiting lysine-specific demethylase 1. EMBO J 2010; 29:1803-1816.

35 Schulte JH, Lim S, Schramm A, et al. Lysine-specific demethylase 1 is strongly expressed in poorly differentiated neuroblastoma: implications for therapy. Cancer Res 2009; 69:2065-2071.

36 Forneris F, Binda C, Adamo A, Battaglioli E, Mattevi A. Structural basis of LSD1-CoREST selectivity in histone $\mathrm{H} 3$ recognition. J Biol Chem 2007; 282:20070-20074.

37 Stavropoulos P, Blobel G, Hoelz A. Crystal structure and mechanism of human lysine-specific demethylase-1. Nat Struct Mol Biol 2006; 13:626-632.

38 Yang M, Gocke CB, Luo X, et al. Structural basis for CoREST-dependent demethylation of nucleosomes by the human LSD1 histone demethylase. Mol Cell 2006; 23:377-387.

39 Chen Y, Yang Y, Wang F, et al. Crystal structure of human histone lysine-specific demethylase 1 (LSD1). Proc Natl Acad Sci USA 2006; 103:13956-13961.

40 Shi YJ, Matson C, Lan F, Iwase S, Baba T, Shi Y. Regulation of LSD1 histone demethylase activity by its associated factors. Mol Cell 2005; 19:857-864.

41 Forneris F, Binda C, Vanoni MA, Battaglioli E, Mattevi A. 
Human histone demethylase LSD1 reads the histone code. $J$ Biol Chem 2005; 280:41360-41365.

42 Forneris F, Binda C, Vanoni MA, Mattevi A, Battaglioli E. Histone demethylation catalysed by LSD1 is a flavin-dependent oxidative process. FEBS Lett 2005; 579:2203-2207.

43 Lee MG, Wynder C, Bochar DA, Hakimi MA, Cooch N, Shiekhattar R. Functional interplay between histone demethylase and deacetylase enzymes. Mol Cell Biol 2006; 26:63956402.

44 Choi HJ, Huber AH, Weis WI. Thermodynamics of betacatenin-ligand interactions: the roles of the $\mathrm{N}$ - and C-terminal tails in modulating binding affinity. J Biol Chem 2006; 281:1027-1038.

45 Huber AH, Weis WI. The structure of the beta-catenin/Ecadherin complex and the molecular basis of diverse ligand recognition by beta-catenin. Cell 2001; 105:391-402.

46 Lickert H, Bauer A, Kemler R, Stappert J. Casein kinase II phosphorylation of E-cadherin increases E-cadherin/betacatenin interaction and strengthens cell-cell adhesion. $J$ Biol Chem 2000; 275:5090-5095.

47 Gessert S, Kuhl M. The multiple phases and faces of wnt signaling during cardiac differentiation and development. Circ Res; 107:186-199.

48 Ciccone DN, Su H, Hevi S, et al. KDM1B is a histone H3K4 demethylase required to establish maternal genomic imprints. Nature 2009; 461:415-418.

49 Bao Y, Hudson QJ, Perera EM, et al. Expression and evolutionary conservation of the tescalcin gene during development. Gene Expr Patterns 2009; 9:273-281.

50 Gutierrez-Ford C, Levay K, Gomes AV, et al. Characterization of tescalcin, a novel EF-hand protein with a single $\mathrm{Ca} 2+-$ binding site: metal-binding properties, localization in tissues and cells, and effect on calcineurin. Biochemistry 2003; 42:14553-14565.

51 Mailander J, Muller-Esterl W, Dedio J. Human homolog of mouse tescalcin associates with $\mathrm{Na}(+) / \mathrm{H}(+)$ exchanger type1. FEBS Lett 2001; 507:331-335.

52 Zaun HC, Shrier A, Orlowski J. Calcineurin B homologous protein 3 promotes the biosynthetic maturation, cell surface stability, and optimal transport of the $\mathrm{Na}+/ \mathrm{H}+$ exchanger NHE1 isoform. J Biol Chem 2008; 283:12456-12467.

53 Li X, Karki P, Lei L, Wang H, Fliegel L. Na+/H+ exchanger isoform 1 facilitates cardiomyocyte embryonic stem cell differentiation. Am J Physiol Heart Circ Physiol 2009; 296:H159-170.

54 Santiago-Martinez E, Soplop NH, Patel R, Kramer SG. Repulsion by Slit and Roundabout prevents Shotgun/E-cadherinmediated cell adhesion during Drosophila heart tube lumen formation. J Cell Biol 2008; 182:241-248.

55 Olson EN, Srivastava D. Molecular pathways controlling heart development. Science 1996; 272:671-676.

56 Burroughs CL, Watanabe M, Morse DE. Distribution of the neural cell adhesion molecule (NCAM) during heart development. J Mol Cell Cardiol 1991; 23:1411-1422.

57 Imanaka-Yoshida K, Matsumoto K, Hara M, Sakakura T, Yoshida T. The dynamic expression of tenascin- $\mathrm{C}$ and tenas-
cin-X during early heart development in the mouse. Differentiation 2003; 71:291-298.

58 Luo Y, Ferreira-Cornwell M, Baldwin H, et al. Rescuing the $\mathrm{N}$-cadherin knockout by cardiac-specific expression of $\mathrm{N}$ - or E-cadherin. Development 2001; 128:459-469.

59 Medrek C, Landberg G, Andersson T, Leandersson K. Wnt$5 \mathrm{a}-\mathrm{CKI}\{$ alpha $\}$ signaling promotes $\{$ beta $\}$-catenin/E-cadherin complex formation and intercellular adhesion in human breast epithelial cells. J Biol Chem 2009; 284:10968-10979.

60 Buchou T, Vernet M, Blond O, et al. Disruption of the regulatory beta subunit of protein kinase CK2 in mice leads to a cell-autonomous defect and early embryonic lethality. Mol Cell Biol 2003; 23:908-915.

61 Blond O, Jensen HH, Buchou T, Cochet C, Issinger OG, Boldyreff B. Knocking out the regulatory beta subunit of protein kinase CK2 in mice: gene dosage effects in ES cells and embryos. Mol Cell Biochem 2005; 274:31-37.

62 Lou DY, Dominguez I, Toselli P, Landesman-Bollag E, O'Brien C, Seldin DC. The alpha catalytic subunit of protein kinase CK2 is required for mouse embryonic development. Mol Cell Biol 2008; 28:131-139.

63 Serres M, Filhol O, Lickert H, et al. The disruption of adherens junctions is associated with a decrease of E-cadherin phosphorylation by protein kinase CK2. Exp Cell Res 2000; 257:255-264.

64 Thienpont B, Zhang L, Postma AV, et al. Haploinsufficiency of TAB2 causes congenital heart defects in humans. Am J Hum Genet; 86:839-849.

65 Biben C, Weber R, Kesteven S, et al. Cardiac septal and valvular dysmorphogenesis in mice heterozygous for mutations in the homeobox gene Nkx2-5. Circ Res 2000; 87:888-895.

66 Bruneau BG, Nemer G, Schmitt JP, et al. A murine model of Holt-Oram syndrome defines roles of the T-box transcription factor Tbx 5 in cardiogenesis and disease. Cell 2001; 106:709721.

67 McFadden DG, Barbosa AC, Richardson JA, Schneider MD, Srivastava D, Olson EN. The Hand1 and Hand2 transcription factors regulate expansion of the embryonic cardiac ventricles in a gene dosage-dependent manner. Development 2005; 132:189-201.

68 Lickert H, Takeuchi JK, Von Both I, et al. Baf60c is essential for function of BAF chromatin remodelling complexes in heart development. Nature 2004; 432:107-112.

69 Gottlieb PD, Pierce SA, Sims RJ, et al. Bop encodes a muscle-restricted protein containing MYND and SET domains and is essential for cardiac differentiation and morphogenesis. Nat Genet 2002; 31:25-32.

70 Montgomery RL, Davis CA, Potthoff MJ, et al. Histone deacetylases 1 and 2 redundantly regulate cardiac morphogenesis, growth, and contractility. Genes Dev 2007; 21:17901802.

71 Lohmann F, Loureiro J, Su H, et al. KMT1E mediated H3K9 methylation is required for the maintenance of embryonic stem cells by repressing trophectoderm differentiation. Stem Cells 2010; 28:201-212.

(Supplementary information is linked to the online version of the paper on the Cell Research website.) 


\section{Retraction: Defective heart development in hypomorphic LSD1 mice}

Thomas B Nicholson, Hui Su, Sarah Hevi, Jing Wang, Jeff Bajko, Mei Li, Reginald Valdez, Joseph Loureiro, Xiaodong Cheng, En Li, Bernd Kinzel, Mark Labow, Taiping Chen

Cell Research advance online publication 10 April 2012; doi:10.1038/cr.2012.60

Retraction to: Cell Research advance online publication 6 December 2011; doi:10.1038/cr.2011.194

The authors unanimously wish to retract this paper because the calcium/calmodulin-dependent protein kinase 2 (CamKII) was erroneously equated with CK2 (Casein Kinase 2) throughout the paper. While all the results were valid and supported the major conclusions, the confusion between CamKII and CK2 led to misinterpretation of some data. Specifically, the speculation of a possible link between CK2 upregulation and E-cadherin hyperphosphorylation in the LSD1-hypomorphic hearts lacked experimental support. This article was only an Advanced Online Publication. The authors apologize for any inconvenience this error may have caused. 